\title{
Pensar los alfabetismos transmedia dentro del aula. Tensiones entre la lógica escolar y las prácticas juveniles
}

\author{
Esteban Ismael Bordón \\ Universidad Nacional de Salta. \\ bordon.ismael@gmail.com \\ ORCID: https://orcid.org/0000-0003-0069-9184 \\ Fecha de finalización: 27 de agosto de 2021 \\ Recibido: 28 de agosto de 2021. \\ Aceptado: 11 de noviembre de 2021. \\ DOI: https://doi.org/10.26422/aucom.2021.1002.bor
}

\begin{abstract}
Resumen
A partir de la irrupción de las tecnologías dentro de las sociedades, las prácticas de los actores sociales se han ido modificando notablemente, por lo que las instituciones educativas no escapan a esto. Dentro del espacio escolar, han persistido a lo largo del tiempo diversas discusiones sobre la implementación de las tecnologías en los procesos educativos, algo que ha dejado entrever la existencia de un desfasaje constante entre los usos de las tecnologías que se dan en el ámbito social y en el ámbito educativo (Martín-Barbero, 2009). En este sentido, el alfabetismo transmedia se enfoca en los usos y prácticas de las tecnologías digitales que los jóvenes desarrollan en su día a día para así poder aprovecharlas dentro de las aulas, dando cuenta de diferentes estrategias de aprendizaje informal que les permite desarrollar una serie de competencias transmedia, que comprende aquellas habilidades que tienen los jóvenes y les permiten participar en los entornos digitales. Esta investigación exploratoria busca analizar aquellas estrategias de aprendizaje que ponen en juego estudiantes de quinto año del Instituto de Educación Media "Dr. Arturo Oñativia” de la ciudad de Salta. A partir de la utilización de encuestas, de la realización de talleres de producción y de entrevistas en profundidad, se indagó sobre los usos y prácticas que los estudiantes tienen de las tecnologías digitales, tanto en sus vidas cotidianas como en las aulas. Los resultados muestran que existe una distancia entre la lógica escolar y las prácticas juveniles, que giran en torno al poco aprovechamiento del celular y de las estrategias de aprendizaje informal que se encontraron en las actividades escolares. Se concluye que se vuelve necesario promover espacios de producción y participación digital en el ámbito escolar, para que los estudiantes puedan desarrollar y hacer uso de las competencias transmedia que poseen.

Palabras clave: alfabetismo transmedia, jóvenes, usos de TIC, educación.

\section{Transmedia literacy in the classroom: tensions between school logic and youth practices}

Abstract

As new technologies break into the classroom, social actors are significantly changing their practices. Educational institutions are not an exception to this trend. For some time now, there have 
been ongoing discussions regarding how best to implement new technologies in educational processes. And these discussions have only revealed the mismatch between how these technologies are used in social spaces and how they are integrated into educational contexts (MartínBarbero, 2009). In this sense, transmedia literacy focuses on the uses and practices of digital technology that young students are developing on a daily basis - uses and practices that can be leveraged in a classroom setting. Through various informal learning strategies, students acquire the transmedia competences that they use to participate in digital environments. This exploratory investigation seeks to analyze the learning strategies employed by fifth-year students at the Dr. Arturo Oñativia High School in the city of Salta, Argentina. Through surveys, production workshops, and in-depth interviews, we looked at the uses and practices of digital technology that students carried out during their daily lives and in the classroom. Our results reveal the gap that exists between school logic and youth practices. In particular, we found that smartphones and informal learning strategies were underutilized in school activities. We conclude that it is necessary to promote spaces for digital production and participation within a school context, so students can develop and make use of the transmedia competences they already have.

Keywords: transmedia literacy, youth, uses of ICTs, education.

\section{Pensando nos letramentos transmídia em sala de aula. Tensões entre a lógica escolar e práticas juvenis}

\section{Resumo}

A partir da irrupção das tecnologias dentro das sociedades, as práticas dos atores sociais foram modificando-se notavelmente, de modo que as instituições educacionais não escapam disso. No espaço escolar, várias discussões sobre a implementação de tecnologias nos processos educacionais têm persistido ao longo do tempo, o que tem sugerido a existência de um distanciamento constante entre os usos das tecnologias que ocorrem na esfera social e social (Martín-Barbero, 2009). Nesse sentido, a alfabetização transmídia foca nos usos e práticas das tecnologias digitais que os jovens desenvolvem no seu dia a dia, a fim de aproveitá-las em sala de aula. Percebe diferentes estratégias de aprendizagem informal que lhes permitem desenvolver uma série de habilidades transmídia, que são aquelas habilidades que os jovens colocam em prática quando participam de ambientes digitais. Esta pesquisa exploratória busca analisar as estratégias de aprendizagem colocadas em prática por alunos do quinto ano do "Dr. Arturo Oñativia" da cidade de Salta. Por meio de pesquisas, realização de oficinas de produção e entrevistas em profundidade, investigou-se os usos e as práticas que os alunos têm das tecnologias digitais, tanto no cotidiano como na sala de aula. Os resultados mostram que existe um distanciamento entre a lógica escolar e as práticas juvenis, que giram em torno do baixo uso do celular e das estratégias de aprendizagem informal encontradas nas atividades escolares. Conclui-se que se torna necessária a promoção de espaços de produção e participação digital no ambiente escolar, para que os alunos possam desenvolver e fazer uso das habilidades transmídia que possuem.

Palavras chave: alfabetização transmedia, jovens, utilizações de TIC, educação.

\section{Introducción}

La irrupción de las tecnologías de la información y la comunicación (TIC) en la vida cotidiana de las personas ha provocado cambios significativos en los entornos culturales de las sociedades (Jenkins, 2010). A partir de los avances tecnológicos que se 


\section{Austral @municación \\ Volumen 10, número 2 (Diciembre de 2021): 375-394 ISSN (I) 2313-9129. ISSN (E) 2313-9137}

van sucediendo, podemos comprender parte de las reconfiguraciones sociales que se producen. Este escenario ha ido complejizando las formas de entender las relaciones entre las TIC y las distintas sociedades, desde definirnos como receptores pasivos de los medios masivos de comunicación a ser prosumidores de contenido dentro de la ecología de medios actual (Scolari, 2015).

Podemos decir, entonces, que las TIC han modificado de distintas maneras los procesos de socialización de los distintos actores sociales. La escuela, como primera institución de socialización secundaria, también ha experimentado cambios importantes como consecuencia de la aparición y uso de las TIC dentro y fuera de los centros (Bernete, 2010). Tras las primeras discusiones en torno a esta cuestión, que se dieron con el debate entre tecnófobos y tecnófilos (Buckingham, 2008; Huergo y Fernández, 2000), las tecnologias se fueron introduciendo en el aula, partiendo desde un enfoque instrumentalista hacia "una visión de comprensión más global de las tecnologías, como fenómeno que atraviesa nuestra configuración de la realidad y de nuestra cultura" (Puggi, 2020, p. 4), lo que supuso una superación a la mirada instrumental de la tecnologia para entender su dimensión estructural de las sociedades contemporáneas como mediación cultural (Martín-Barbero, 2009).

Si bien las instituciones educativas han ido adoptando las tecnologías dentro del aula, el sistema escolar siempre mantuvo distancia con el sistema comunicativo de la sociedad. Podemos mencionar dos procesos que pueden ayudar a explicar esta situación. El primero refiere al descentramiento y diseminación de los saberes por fuera de la institución escolar (Martín-Barbero, 2009), lo que determina una transformación en los modos de circulación del saber que históricamente le pertenecieron al sistema educativo. El segundo se corresponde con las estrategias para incorporar las TIC dentro del aula, que distan demasiado de las formas en que los jóvenes se vinculan con las tecnologías en su vida cotidiana, ignorando procesos de apropiación social de las TIC y exponiendo "un desfasaje evidente entre el uso que de las TIC se hace a nivel general y el que se realiza en las escuelas" (Almirón, 2014, p. 4).

En este trabajo presentamos algunos de los principales resultados de la investigación "Competencias transmedia de los jóvenes salteños: Diálogos entre prosumidores y escuela", en el que indagamos sobre las prácticas y usos de las tecnologías digitales que tienen los estudiantes de un colegio secundario de la provincia de Salta. A partir de la realización y análisis de entrevistas en profundidad y talleres de producción, encontramos distintos elementos que nos permiten comprender el desfasaje que men-

Proyecto de investigación realizado en el marco de una Beca Estímulo a las Vocaciones Científicas, del Consejo Interuniversitario Nacional. Asimismo, el estudio se inserta dentro del proyecto de investigación $\mathrm{N}^{\circ} 2681 / 0$ del CIUNSA, titulado "Nuevos Formatos Discursivos: Periodismo Digital y Narrativas Transmedia", dirigido por el Mg. Sergio Grabosky. 
Esteban Ismael Bordón

Pensar los alfabetismos transmedia dentro del aula. Tensiones entre la lógica escolar y las prácticas juveniles

cionamos, entre la lógica escolar y la mirada joven, al integrar las tecnologías digitales dentro del aula desde el enfoque del alfabetismo transmedia.

\section{Jóvenes prosumidores y alfabetismos transmedia}

En la actualidad existe una multiplicidad de estudios en torno a la categorización de los jóvenes, lo que nos brinda una variedad de perspectivas para su abordaje. En este trabajo discutimos con aquellos enfoques biologicistas que sitúan a la juventud como un proceso delimitado por la edad (Dávila León, 2004), entre la niñez y la adultez. Asimismo, nos distanciamos de la concepción de la juventud como una moratoria social, que implica la postergación de formar una familia o ingresar al mercado laboral puesto que, aunque introduce una diferenciación social de la juventud, "sería una característica reservada para sectores sociales con mayores posibilidades económicas" (Margulis y Urresti, 1998).

Desde una perspectiva sociocultural, al pensar en la categoría de jóvenes, Rossana Reguillo (2003) lo problematiza como una construcción sociohistórica, ya que "las distintas sociedades en diferentes etapas históricas han planteado las segmentaciones sociales por grupos de edad de muy distintas maneras y que, incluso, para algunas sociedades este tipo de recorte no ha existido" (p. 104). Desde este enfoque, la antropóloga mexicana considera a los jóvenes como sujeto social que

Constituyen un universo social, cambiante y discontinuo, cuyas características son resultado de una negociación-tensión entre la categoría sociocultural asignada por la sociedad en particular y la actualización subjetiva que sujetos concretos llevan a cabo a partir de la interiorización diferenciada de los esquemas de la cultura vigentes. (Reguillo, 2003, p. 50)

En este sentido, proponemos pensar la categoría de jóvenes sin agotarla o reducirla a condiciones tales como la edad biológica, el ser estudiantes o la inserción socioeconómica, sino considerar la constitución cultural que es propia de cada sociedad y reconocerla como una categoría heterogénea, dinámica y discontinua históricamente (Reguillo, 2003). Creemos, desde esta mirada, que la apropiación y los usos sociales de las tecnologías se hace más claramente visible en los jóvenes, en sus empatías cognitivas y expresivas con las tecnologías y en los nuevos modos de percibir el espacio y el tiempo, la velocidad y la lentitud, lo lejano y lo cercano (Martín-Barbero, 2006), por lo que conforma una dimensión relevante en la definición de lo que significa ser joven en la sociedad actual.

Partimos de considerar a los jóvenes como prosumidores, en tanto nos ayuda a pensar en las múltiples formas que tienen de relacionarse con las tecnologías digitales. Las formas de construir el sentido social se han modificado, la distancia entre producir y consumir se ha reducido al punto de podernos considerar productores y 


\section{Austral @municación \\ Volumen 10, número 2 (Diciembre de 2021): 375-394 ISSN (I) 2313-9129. ISSN (E) 2313-9137}

consumidores (García Canclini, 2007), capaces de utilizar la información producida con un propósito y darle otro. Se lee de otras formas, ahora el lector puede intervenir el texto electrónico, "cortar, desplazar, cambiar el orden, introducir su propia escritura" (Chartier, 1999, p. 205).

Decimos que en la actualidad podemos pensar a los jóvenes desde esta concepción, ya que "la interpenetración de los movimientos de producción, consumo y participación hace que hoy podamos pensarnos como prosumidores" (García Canclini, 2012a, p. 4). Desde este enfoque, los jóvenes dejan de pensarse solamente como audiencias pasivas de los medios, debido a que hoy en día ocupan papeles protagónicos en las industrias audiovisuales y digitales, siendo "los que conducen o participan más creativamente en las tecnologías digitales en casi todas las áreas de la creación y la comunicación cultural" (García Canclini, 2012b, p. 6).

Si bien la categoría conceptual de prosumidor nos ayuda a comprender a los jóvenes como participantes activos en el sistema de comunicación social actual y no como audiencia pasiva, es importante destacar que esta característica no define a los jóvenes, ya que no todos son prosumidores a un mismo nivel y existen procesos de conformación de los sujetos como usuarios de las tecnologías digitales. Por esto, discutimos con las categorías de nativos digitales (Piscitelli, 2009; Prensky, 2001), en tanto limitan la comprensión de los procesos de apropiación social de las tecnologías. Así, pensamos en los procesos de alfabetización en tecnologías como una categoría conceptual que permite indagar la conformación de los jóvenes como prosumidores.

Si hablamos de las relaciones entre jóvenes, escuela y medios, encontramos a la alfabetización en medios -o alfabetización mediática- como uno de los antecedentes iniciales, con origen en Europa y América del Norte (Albarello y Mihal, 2018). En una primera etapa, la alfabetización mediática estuvo centrada en la lectura crítica de los mensajes potencialmente negativos de los medios masivos. En un segundo momento, también se pusieron en marcha propuestas de producción de contenido desde las escuelas, pasando de la lectura a la producción crítica (Albarello y Mihal, 2018; Scolari et al., 2018). Sin embargo, el escenario de medios en el que tuvo origen la alfabetización mediatica se ha ido modificando, por lo cual se hizo necesario actualizar el enfoque desde el que se lo aborda.

Este escenario de medios es posible entenderlo desde la noción de "sociedad red" (Castells, 2005), que da cuenta de una nueva estructura social que tiene como base material a las tecnologías e internet, que se caracteriza a partir de dos procesos: el de la participación de los individuos con intereses similares y una cultura del compartir. Estos procesos permiten entender las nociones de "cultura de la convergencia" y "comunicación transmedia” que propone Henry Jenkins (2010), ya que expone las formas en las que el conocimiento y la información circulan por redes digitales y los diferen- 
Esteban Ismael Bordón

Pensar los alfabetismos transmedia dentro del aula. Tensiones entre la lógica escolar y las prácticas juveniles

tes medios, en los que los usuarios no solamente consumen mensajes, sino también producen, comparten y participan en el sistema de medios. De esta manera, se vuelve necesario tomar los aportes de la teoría de la ecología de medios, en tanto permiten comprender el escenario de medios en el cual se desenvuelven los prosumidores. Siguiendo a Scolari (2015), podemos interpretar la metáfora ecológica de dos formas: 1) considerar a los medios como generadores de ambientes que afectan a los sujetos que la utilizan; 2) considerar a los medios como especies, en los que ningún medio adquiere su significado por sí mismo, sino en procesos de interacción entre sí afectándose entre ellos, por lo cual atraviesan procesos de adaptación, hibridación y coevolución y extinción. De esta forma, podemos comprender el escenario en el cual participan los usuarios, en el que no solo pueden actuar como consumidores, productores y distribuidores del conocimiento y del contenido digital, sino que a través de sus prácticas con y a través de los medios estos adquieren nuevos significados.

Ante este nuevo escenario comunicativo es que Scolari (2018a) propone actualizar la noción de "alfabetización mediática" desde el concepto de "alfabetismo transmedia". Este concepto propone una lectura diferente de la realidad de los jóvenes, considerándolos "personas potencialmente capaces de generar y compartir contenidos de diferentes tipos y niveles de complejidad" (Scolari, 2018c). Partimos de la idea de que no existen nativos digitales, sino que, a través del alfabetismo transmedia, los jóvenes desarrollan diferentes habilidades que les permiten participar dentro del ecosistema de medios. Estas habilidades las reconocemos bajo el concepto de "competencias transmedia”, que se definen como

\footnotetext{
Una serie de habilidades relacionadas con la producción, el intercambio y el consumo de medios interactivos digitales. Estas competencias van desde los procesos de resolución de problemas en videojuegos hasta la producción y el intercambio de contenidos en plataformas web y redes sociales; la creación, producción, intercambio y consumo crítico de contenido narrativo (fanfiction, fanvids, etc.) por los adolescentes también forma parte de este universo. (Scolari, 2018c, p. 17)
}

Entendemos que analizar las competencias transmedia de los jóvenes permite comprender cómo se desarrolla la apropiación social de las tecnologías. Sin embargo, hablar de alfabetización nos remite a los procesos de enseñanza-aprendizaje formal e intencionado, mientras que "los alfabetismos ocurren de forma inesperada como resultado de prácticas sociales y culturales que pueden (o no) detonar aprendizajes significativos" (Corona Rodríguez, 2018, p. 140). Es por esto que decidimos hablar de alfabetismos y no de alfabetización transmedia, ya que los procesos y estrategias de aprendizaje que tienen los jóvenes para con las tecnologías digitales están sucediendo fuera de las aulas y son diversos, por lo que buscamos conocer qué hacen los jóvenes 
con los medios en su vida cotidiana, para así encontrar formas de incluir estas prácticas de aprendizaje y participación en los medios dentro de las escuelas.

\section{Estrategias para abordar los alfabetismos transmedia de jóvenes prosumidores}

El objetivo general de esta investigación es indagar las estrategias de aprendizaje que jóvenes estudiantes, a los cuales consideramos prosumidores, desarrollan fuera del aula y las formas en las que la institución educativa las toma en cuenta en sus clases. En este sentido nos preguntamos: ¿qué hacen los jóvenes con los medios?, ¿cómo aprenden a hacer lo que hacen con los medios?, ¿de qué forma se integran estas competencias y estrategias dentro de las aulas? Consideramos, de esta forma, que los jóvenes no son nativos digitales, sino que existen procesos de aprendizaje que permiten desarrollar competencias transmedia y que se relacionan con los accesos, usos e intereses que tienen de las tecnologías y los medios digitales, lo que lleva a conformarlos como prosumidores.

Para cumplir con los objetivos planteados, y entendiendo que es un tema poco estudiado en la región, específicamente en la provincia de Salta se llevó adelante una investigación de tipo exploratoria, con un sustento epistemológico en el paradigma interpretativo, el cual postula que existen múltiples realidades construidas por los actores en su relación con la realidad social en la cual viven (Martínez Rodríguez, 2011). Por ello, esta investigación se ubica en un enfoque cualitativo, en tanto proporciona conocimiento acerca de la dinámica de los procesos sociales, del cambio y del contexto social (Mason, 2006), lo que nos posibilita abordar los procesos de alfabetismos transmedia que atraviesan los jóvenes. En este trabajo, hemos apostado por aplicar el enfoque etnográfico para el estudio de un caso (André, 1998), que involucra el estudio profundo de una entidad singular a través la observación participante, trabajo de campo e interacción personal con los sujetos; en tanto, el diseño metodológico de tipo etnográfico se reconoce como una metodología adecuada para los estudios de jóvenes, nuevos medios y educación (Winocur et al., 2018).

Para la realización de esta investigación se contó con la participación anónima de alumnos pertenecientes al quinto año del Instituto de Educación Media "Dr. Arturo Oñativia" (IEM), de la provincia de Salta, en el espacio curricular de la materia Idioma Nacional y Literatura. Este colegio tiene la particularidad de ser un centro de educación secundaria perteneciente a la Universidad Nacional de Salta, al que concurren jóvenes de distintos puntos de la ciudad. Dentro del contexto de pandemia por COVID-19, se trabajó con seis grupos burbujas de aproximadamente quince estudiantes cada uno.

Para la recolección de datos, en esta investigación apostamos por la utilización de tres estrategias: 1) un cuestionario inicial sobre contextos socioculturales, usos y prácticas de los medios y tecnologías digitales; 2) talleres participativos para explorar 
Esteban Ismael Bordón

Pensar los alfabetismos transmedia dentro del aula. Tensiones entre la lógica escolar y las prácticas juveniles

las prácticas transmedia de los jóvenes; 3) la realización de entrevistas en profundidad con informantes clave. Todos los encuentros fueron presenciales, a excepción de un taller de planificación de podcast que se realizó en modalidad virtual. ${ }^{2}$

La realización de un cuestionario inicial tuvo el objetivo de obtener información general sobre la relación que tienen los jóvenes con las tecnologías digitales. En un primer apartado, les consultamos a los estudiantes sobre datos sociodemográficos relacionados con el acceso a las tecnologías que tienen en sus vidas cotidianas. En el resto de apartados, se les consultó sobre sus conocimientos, motivaciones, usos y prácticas de dispositivos y tecnologías digitales vinculadas a los textos, imágenes, audios, videos, videojuegos y redes sociales en entornos digitales. Por último, se les consultó sobre las formas en que creen que se aprovechan y hacen usos de sus habilidades digitales dentro de sus actividades escolares.

Por su parte, la realización de talleres participativos de producción fue una decisión metodológica pensada específicamente para el fenómeno que abordamos. En este sentido, recurrimos a la etnografía digital como enfoque de investigación, en tanto es adecuada para este tipo de estudios, ya que según Pink y Ardevol (2018):

\begin{abstract}
Es una estrategia de investigación que se centra en revelar cosas de las que normalmente no se habla (por ejemplo, las estrategias de aprendizaje y las habilidades que los adolescentes ponen en juego cuando usan las tecnologías digitales en casa), y aspectos sensoriales, elementos emocionales y habituales de la vida cotidiana que las personas no suelen mostrar a los demás (por ejemplo, las rutinas cotidianas que tienen los adolescentes cuando se levantan por la mañana, llegan a casa y antes de acostarse en relación a ver películas o series, contarse historias o chismes a través de las tecnologías cotidianas). (p. 114)
\end{abstract}

Es decir, consideramos que para explorar las competencias transmedia y las estrategias de aprendizaje informales que los jóvenes utilizan y desarrollan en su día a día no alcanzaba solo con diálogos en profundidad con los participantes, sino que surgió la necesidad de crear espacios de producción transmedia para revelar motivaciones, prácticas y usos cotidianos de las tecnologías y medios digitales. Así, el enfoque etnográfico resulta fundamental, en tanto ofrece la posibilidad de observar los procesos de los estudiantes en un entorno de prácticas y usos de las tecnologías y medios digitales que, a través de encuestas o entrevistas, no podrían surgir, tanto por ser prácticas naturalizadas como cotidianas. En este escenario, la capacidad de observación y análisis del investigador se vuelve fundamental para reconocer e identificar las diferentes competencias y estrategias de aprendizaje informal que ponen en juego los estudiantes. La realización de los talleres tuvo una duración de dos horas semanales a lo largo de tres meses, con encuentros quincenales debido a la disposición de burbujas sanitarias, trabajando así con seis grupos de estudiantes.

Taller realizado en modalidad virtual el 8 de julio de 2021 por la plataforma Zoom. 


\section{Austral Comunicación \\ Volumen 10, número 2 (Diciembre de 2021): 375-394 ISSN (I) 2313-9129. ISSN (E) 2313-9137}

La propuesta de talleres de producción de podcasts nace a partir de reconocerla como una estrategia provechosa para los objetivos de nuestra investigación, ya que conlleva la posibilidad de trabajar con múltiples dispositivos y la oportunidad de poder observar el desarrollo de estrategias de aprendizaje informal, en tanto los estudiantes no estaban tan familiarizados con el consumo de podcasts ni la edición de audio. Así, los docentes del departamento de Idioma Nacional y Literatura nos abrieron las puertas para trabajar en sus espacios y horarios de clases. ${ }^{3} \mathrm{Al}$ sumarnos a las clases de una materia específica, debimos adaptarnos a los contenidos que se estaban trabajando, que fue la lectura de la novela La increible y triste historia de la Cándida Eréndira $y$ de su abuela desalmada de Gabriel García Márquez. A partir de encontrarnos en este contexto, se les propuso a los estudiantes la producción de un podcast breve sobre algún tema que tratara la novela, brindándoles la libertad de que pudieran elegir aspectos que fueran interesantes para ellos, como también para la elección de los dispositivos con los que trabajarían. Nuestro papel como investigadores consistió en la observación participante, lo cual nos brindó la posibilidad de interactuar con los alumnos y observar tanto las estrategias de producción y aprendizaje informal como las dudas y certezas que ellos evidenciaron. Los resultados finales de los talleres fueron de dos producciones: la escritura de un guion y la creación de un podcast, cuya duración fue elegida por ellos mismos y que rondaron desde tres hasta quince minutos.

Por último, se llevaron a cabo entrevistas en profundidad con informantes clave. Estos fueron seleccionados a partir de las observaciones realizadas durante los talleres, siendo elegidos aquellos estudiantes que mostraron usos y prácticas interesantes para nuestra investigación. Entre ellos encontramos jóvenes que desconocían prácticas de edición y producción de audios, otros con competencias avanzadas sobre el uso de audio digital y participantes que propusieron estrategias novedosas para la producción del podcast, entre otros. Las entrevistas realizadas fueron semiestructuradas, con temáticas centrales relacionadas con las competencias transmedia sobre los usos de audio digital, las estrategias de aprendizaje informal desarrolladas durante los talleres y el uso de sus competencias y estrategias de aprendizaje dentro de las aulas.

Para abordar el análisis de los datos recolectados, partimos desde el método de la teoría fundamentada (Strauss y Corbin, 2002), que se refiere a una teoría derivada de datos recopilados de manera sistemática y analizados por medio de un proceso de investigación, en el que la recolección de datos, el análisis y la teoría que surgirá de ellos guardan estrecha relación entre sí. Una de las estrategias principales para el desarrollo de la teoría fundamentada se basa en el método comparativo constante, que es un modo de generar teoría a partir del análisis comparativo de los datos, el cual requiere la

Las clases de Idioma Nacional y Literatura, del quinto año del IEM, tienen una carga horaria de un módulo completo los días miércoles y medio módulo los días jueves, con un total de dos horas reloj a la semana. 
Esteban Ismael Bordón

Pensar los alfabetismos transmedia dentro del aula. Tensiones entre la lógica escolar y las prácticas juveniles

saturación de los datos y facilita la generación de categorías a partir de la comparación entre hechos similares o diferentes (Cuñat Giménez, 2007).

Como corpus de análisis se trabajó con información recogida con los cuestionarios iniciales, con registros de observación realizados durante el desarrollo de los talleres y con transcripciones de las entrevistas en profundidad. A partir de la lectura de estos documentos, se realizó el análisis manual de los resultados siguiendo la propuesta de Valles Martínez (1999), que consiste en la codificación, clasificación e integración como etapas de tratamiento analítico del corpus, a partir de un análisis centrado en temas relacionados con el aprovechamiento de las competencias transmedia en las aulas.

\section{Distancias y encuentros entre la escuela y los jóvenes}

Los hallazgos de esta investigación se presentarán a partir de las secciones temáticas que fueron surgiendo en el análisis de los datos obtenidos. Así, encontramos tres apartados temáticos que ayudan a comprender parte del desfasaje que existe entre la lógica escolar, las estrategias de consumo/creación transmedia y las prácticas de los jóvenes al relacionarse con tecnologías y medios digitales en sus vidas cotidianas.

\section{1) Usos de tecnologías y medios digitales por parte de jóvenes}

Al buscar comprender las prácticas que los jóvenes tienen de las tecnologías y los medios digitales es necesario tener en cuenta cuáles son aquellos dispositivos que utilizan. En este sentido, los estudiantes del IEM mencionaron, a través de una encuesta, que el smartphone es el dispositivo que más utilizan, seguido por la computadora de escritorio y los ordenadores portátiles, mientras que Instagram es la red social más usada, seguida por WhatsApp, tanto para informarse (Bordón, 2020) como para estudiar, entretenerse y comunicarse.

Los dispositivos móviles nos permiten acercarnos a las prácticas de los estudiantes en la actualidad, en las que prevalece una cultura móvil juvenil (Castells et al., 2007) que define y moldea las formas en las que los jóvenes se relacionan con y a través de las tecnologías digitales. El celular, o smartphone, se vuelve un elemento primordial para entender las prácticas de los jóvenes en su día a día, debido a que se convierte en un dispositivo que pueden llevar a cualquier lado y, con la posibilidad que brinda la conectividad móvil, les permite permanecer conectados a sus redes. Durante la planificación de los talleres, se había pensado en crear un espacio de producción de podcast con netbooks en las aulas o con computadoras de escritorio en la sala de informática del Instituto, pero al ir acercándonos a los usos y prácticas que tienen los estudiantes del IEM, fuimos modificando esta propuesta, dado que la gran mayoría nos mencionó el interés por trabajar con el celular en lugar de un ordenador. Un grupo reducido de 


\title{
Austral Comunicación \\ Volumen 10, número 2 (Diciembre de 2021): 375-394 ISSN (I) 2313-9129. ISSN (E) 2313-9137
}

estudiantes solicitó trabajar con computadoras, debido a la complejidad de producción con la que se propusieron trabajar, por ejemplo, al realizar un podcast de conversación y opinión sobre la novela utilizando la plataforma Discord ${ }^{4}$ y el programa OBS Studio ${ }^{5}$ para la grabación (Benjamín, Gonzalo, Ricardo y Tomás, estudiantes de quinto año en el IEM, comunicación personal, 18 de agosto de 2021).

En cuanto a las redes sociales más utilizadas, también brindan un pantallazo de los consumos digitales actuales, siendo Instagram la red preferida por los jóvenes. En esta red social predominan los recursos audiovisuales por sobre cualquier otro y pasan allí gran parte de sus ratos libres. El interés de los estudiantes en su cotidianeidad en la red pasa por mirar publicaciones de los usuarios que siguen y navegar por la sección explore, que presenta publicaciones en tendencia basados en intereses personales. Sin embargo, es muy poco frecuente la publicación propia y, si lo hacen, prefieren publicar en sus stories antes que en su feed.

\footnotetext{
[¿Qué es lo que más ocupa tu tiempo?] Y... en Instagram, veo cosas, pero no publico nada. [¿Y qué solés ver?] De todo un poco me sale, ya sea frases o posteos de los chicos, memes, un poco de todo. (Milagros, estudiante de quinto año en IEM, comunicación personal, 10 de junio de 2021)
}

Sus prácticas de producción, además, están muy vinculadas con lo que ofrece la aplicación Instagram, debido a que reconocen saber poco sobre edición de imágenes o de videos, por el contrario, prefieren utilizar herramientas predefinidas en la aplicación:

\begin{abstract}
[¿Usás Instagram?] Sí, sí, pero no subo fotos, subo historias y las destaco sino. No estoy segura de subir publicaciones todavía, pero después sí subo historias de mi coneja, de viajes. [¿Y hacés ediciones o grabás y lo subís así nomás?] No, porque ya vienen filtros en Instagram. Le cambio el color y esas cosas. (Agostina, estudiante de quinto año en el IEM, comunicación personal, 10 de junio de 2021)
\end{abstract}

A partir de reconocer el celular y lo visual como elementos -más- preponderantes en la configuración de las relaciones que establecen los jóvenes con las tecnologías digitales, podemos pensar en cómo la cultura móvil y la abundancia de lo visual caracterizan las prácticas y los intereses que tienen los estudiantes dentro del ecosistema mediático en la actualidad. Así, también se hace notoria la preferencia de la lectura/ consumo por sobre la escritura/producción, dado que demuestran pasar mucho tiempo navegando en redes sociales sin tener la necesidad de publicar, algo que hacen en

\footnotetext{
Es una aplicación multiplataforma de mensajería instantánea que ofrece los servicios de llamadas de voz y video, además de chat por textos. Está orientado a la comunicación de usuarios en el ámbito gamer y suele utilizarse al jugar videojuegos cooperativos.

5 Es una aplicación multiplataforma para la grabación y transmisión de video por internet. Está orientado a la emisión en directo en plataformas de streaming.
} 
Esteban Ismael Bordón

Pensar los alfabetismos transmedia dentro del aula. Tensiones entre la lógica escolar y las prácticas juveniles

muy pocas ocasiones. La gran mayoría de las actividades cotidianas de los estudiantes están atravesadas por la pantalla de sus teléfonos celulares, ya sea al informarse, entretenerse o comunicarse, en las que este dispositivo toma el lugar de aparato metamedium (Manovich, 2013), ya que ofrece la posibilidad de acceder a diversas plataformas, medios y aplicaciones en todo momento y lugar.

\section{2) Estrategias de aprendizaje informal de los estudiantes}

Los usos y prácticas que los jóvenes tienen con las tecnologías digitales fuera de las aulas constituye una experiencia educativa compleja, lo que nos invita a considerar otras formas de aprendizaje y apropiación de las tecnologías por parte de los estudiantes, como también a tener en cuenta los procesos de prosumición que llevan adelante en sus prácticas con las TIC. Uno de los objetivos centrales de la realización de talleres consistía en poder indagar sobre estas estrategias de aprendizaje de los estudiantes, dado que nos brindaba muchas ventajas para conocer en profundidad usos y prácticas de los dispositivos y medios digitales in situ que, con herramientas como las encuestas o las entrevistas, pueden no aparecer tan claramente.

La idea de trabajar en la producción de un podcast nos posibilitó indagar sobre las estrategias de aprendizaje informal que tienen los jóvenes, en tanto no estaban tan familiarizados con este tipo de producción, por lo que la propuesta fue promover un entorno de producción digital para que pudieran poner en juego estas estrategias. Partiendo de una diferenciación con las estrategias de aprendizaje formal que involucran "formas estructuradas, controladas y, a menudo, forzadas que se emplean en el ámbito escolar" (Scolari, 2018b, p. 84), entendemos que las estrategias informales de aprendizaje se presentan de una forma desordenada, sin una lógica del paso a paso, y los conocimientos no se adquieren de forma consciente, sino que surgen de la experiencia con los medios digitales y en vinculación con otros usuarios. Así, durante el trabajo con los estudiantes en los talleres, se detectaron principalmente tres estrategias informales de aprendizaje:

- Aprendizaje mediante el consumo: es una estrategia mediante la cual los jóvenes adquieren conocimientos sobre formatos y géneros de producciones digitales al consumirlos. En el caso del trabajo de los talleres, al presentarles la propuesta, relacionaron el podcast con el género radial, lo que sirvió para pensar el desarrollo de sus producciones:

[¿Conocen lo que es un podcast?] Sí, un podcast es como un programa de radio, pero es todo grabado, o sea, antes hay que editarlo y todo eso. (Santiago, estudiante de quinto año en el IEM, comunicación personal, 10 de junio de 2017) 


\title{
Austral Omunicación \\ Volumen 10, número 2 (Diciembre de 2021): 375-394 ISSN (I) 2313-9129. ISSN (E) 2313-9137
}

Además, ya dentro del género del podcast, señalaron diversos ejemplos que les sirvieron de referencia para la planificación de su propio guion, como el podcast "Historias Innecesarias" de Damián Kuc (Juliana, estudiante de quinto año en el IEM, comunicación personal, 1 de julio de 2021) o TED Talks en español (Mariano, estudiante de quinto año en el IEM, comunicación personal, 1 de julio de 2021).

- Aprendizaje mediante la imitación: el estudiante reproduce acciones de un experto, lo que le permite, además de recordar un conocimiento, aprender nuevas competencias. Este aprendizaje se vincula con la resolución de problemas, en tanto acuden a un experto siempre que se les presente una dificultad al querer realizar alguna actividad. La figura de los tutoriales se vuelve un elemento fundamental para comprender este tipo de aprendizaje:

\begin{abstract}
Yo sé un poco de edición de audio, aunque igual, si no sé algo, me busco un tutorial, que en YouTube hay un montón. (Virginia, estudiante de quinto año en el IEM, comunicación personal, 19 de junio de 2021)
\end{abstract}

Aquí es importante señalar el papel que cumple el celular como metamedium, en tanto no solo les funcionó como dispositivo para la grabación y edición del podcast, sino que también les ofreció la posibilidad de consultar tutoriales de expertos ante cualquier duda que surgió en el proceso.

[¿De qué forma tienen pensado trabajar la grabación y edición?] Con el celular, porque se me hace más cómodo. Yo sé editar videos, uso InShot ${ }^{6}$ en el celular, pero nunca edité audios, aunque creo que será casi igual. Cualquier cosa me miro un tutorial y listo. (Moisés, estudiante del quinto año en el IEM, comunicación personal, 26 de agosto de 2021)

- Aprendizaje mediante la práctica: este tipo de aprendizaje surge en los usos que los jóvenes hacen de las tecnologías, conociendo la forma en que funciona la aplicación o dispositivo mientras se hace uso de ella, mostrando el carácter experimental que brindan las tecnologías digitales. En particular, durante la realización de los talleres, este proceso se detectó mientras llevaban adelante la grabación y edición del podcast, lo que conllevó prácticas de ensayo/error. Al llevar auriculares, los estudiantes tenían la posibilidad de escuchar en simultáneo la edición, proponiendo cambios y mejoras durante el proceso mientras exploraban las posibilidades que les ofrecía la aplicación de creación de podcast.

\footnotetext{
Nosotras nos vamos grabando y vamos viendo cómo sale, cuando algo nos gusta, seguimos, y si no, volvemos a grabar, porque se nos hace más fácil ir improvisando así mientras leemos la novela. Mientras vamos avanzando vamos viendo cómo agregar otras cosas a las voces. (Agostina y Luciana, estudiantes del quinto año en el IEM, comunicación personal, 19 de agosto de 2021)
}

${ }_{6}$ Aplicación de edición para dispositivos con sistema operativo Android. 
Esteban Ismael Bordón

Pensar los alfabetismos transmedia dentro del aula. Tensiones entre la lógica escolar y las prácticas juveniles

\section{3) Usos y prácticas escolares con tecnologías y medios digitales}

Uno de los puntos que más nos señalaron los estudiantes al hablar sobre los usos de dispositivos y medios digitales para sus clases se centraron especialmente en dos elementos: el uso de WhatsApp y la escritura en computadora.

En primer lugar, el uso de WhatsApp se corresponde con la necesidad de que los jóvenes estén conectados con el colegio. Así, esta aplicación cotidiana, tanto para los estudiantes como para los profesores, funcionó y funciona como herramienta clave para el contacto áulico más allá de los espacios y tiempos definidos por la institución. Durante la realización de esta investigación, el uso de esta red social, a través de conformación de grupos realizados previamente con los docentes, nos sirvió como punto de encuentro con los estudiantes fuera de los horarios de los talleres, ya sea para el recordatorio de actividades, evacuar dudas o acercarles información. Por fuera de los talleres, los grupos de WhatsApp fueron fundamentales cuando el dictado de clases presenciales no fue posible durante el aislamiento y distanciamiento social por COVID-19. Otras herramientas digitales que se utilizaron por este mismo motivo fueron la plataforma Zoom y Moodle.

En relación con los usos específicos de las TIC dentro de las aulas, podemos reconocer cómo la cultura lectoescritora sigue muy presente en los espacios educativos formales, migrando del trabajo en papel al uso de dispositivos digitales. Este punto es importante para pensar las brechas entre las prácticas sociales y las escolares de los jóvenes, en tanto relacionan mucho la escritura digital con sus tareas, en las que el procesador de textos Word aparece como la herramienta más importante.

[¿Qué hacés en la computadora por lo general?] Las tareas o ver vídeos, la computadora la uso de vez en cuando nomás, pero más estoy con el teléfono. [¿Y qué tareas te mandan a hacer?] Bueno, todo lo que sea hacer en un archivo Word, todo eso lo hago por computadora. [¿Y en el celular escribís?] Sí, también uso el Word, toda tarea, ja. (Milagros, estudiante de quinto año en el IEM, comunicación personal, 10 de junio de 2021)

La gran mayoría de las actividades escolares que aprovechan los usos y prácticas de las tecnologías se relacionan con el uso de procesadores de texto. En este caso, el uso de la computadora por sobre el celular tiene que ver con decisiones de los propios estudiantes, ya que les resulta mucho más cómodo utilizar el teclado antes que las pantallas táctiles.

[Y la computadora, ¿cuándo tenés que usarla?] Solo la uso para hacer la tarea. [Por ejemplo ¿cuáles?] Bueno, uso el Word porque me parece mucho más cómodo estar en el teclado que con el celular. Y bueno, ahí hago las tareas de todas las materias. (Agostina, estudiante de quinto año en el IEM, comunicación personal, 10 de junio de 2021) 


\section{Austral Omunicación \\ Volumen 10, número 2 (Diciembre de 2021): 375-394 ISSN (I) 2313-9129. ISSN (E) 2313-9137}

La decisión de no utilizar sus celulares para realizar las tareas también la justifican por el rasgo distractor (Albarello, 2018) que puede tener este dispositivo, debido a las funciones que desempeña, apareciendo nuevamente características del metamedium. En este caso, se menciona el uso de una tableta, pero que tiene el mismo sentido -el de relacionar al celular con la conectividad permanente- como distracción para las tareas:

\footnotetext{
[¿Qué hacés con la tableta?] Por ahí hacer trabajos que hay que entregarlos virtual, digamos. A veces ocupo el teléfono y otras veces la tablet, porque es como una distracción, porque ya me empiezan a llegar mensajes y eso. [Entonces, ¿para hacer las tareas preferís usar la tableta?] Sí. (Florencia, estudiante de quinto año en el IEM, comunicación personal, 10 de junio de 2021)
}

Al pensar en las estrategias de aprendizaje informal que desarrollan los jóvenes al hacer uso de las tecnologías y medios digitales se observa otro vacío entre la lógica escolar y las prácticas juveniles. Tanto las estrategias de aprendizaje mediante el consumo, el aprendizaje mediante la imitación y el aprendizaje mediante la práctica nos permiten considerar el potencial abierto y flexible que brindan los entornos digitales y que proceden más allá de las aulas (Clark et al., 2009), rasgos distintos a los que propone el entorno educativo, con formas más estructuradas y controladas. Muchas veces, esta lógica escolar se relaciona con la enseñanza de algún contenido por parte de los profesores y la realización de consignas que demuestran lo aprendido por parte de los estudiantes, cuando se podrían aprovechar las estrategias de aprendizaje que desarrollan los jóvenes en sus entornos informales.

\section{[¿Cómo llevan las tareas de la lectura de la novela?] Más o menos, nos pidieron leer la novela y responder una guía de preguntas, pero no la hicimos todavía porque nos aburre un poco. (Agustín, María y Samuel, estudiantes del quinto año en el IEM, comunicación personal, 1 de julio de 2021)}

Es clave pensar en los procesos de usos de las tecnologías y estrategias de aprendizaje informal que experimentan los jóvenes para comprender este desfasaje entre la lógica escolar y las prácticas juveniles. Los procesos de enseñanza/aprendizaje formales continúan siendo importantes, pero debemos tener en cuenta que los modelos informales adquieren un papel clave en la educación moderna de los jóvenes, en tanto son autodidactas, tienden a conformar comunidades culturales y participan en medios sociales por fuera de las aulas (Black et al., 2015).

\section{Conclusiones y propuestas a futuro}

Los hallazgos de esta investigación nos permiten reflexionar sobre el lugar que tienen los alfabetismos transmedia en las aulas, a partir de las distancias y los encuentros 
Esteban Ismael Bordón

Pensar los alfabetismos transmedia dentro del aula. Tensiones entre la lógica escolar y las prácticas juveniles

que existen entre las prácticas y los usos de las tecnologías digitales que los jóvenes desarrollan tanto en espacios informales como en espacios formales educativos.

La primera cuestión que surge gira en torno a la utilización de los dispositivos en los espacios educativos. El celular ocupa, hoy en día, un lugar preponderante en las vidas de los jóvenes, en tanto funciona como metamedium que posibilita la realización de diversas tareas y de la participación constante en los medios digitales, ya sea como prosumidores de contenidos o como participantes en comunidades virtuales. Por su parte, en los espacios educativos predomina el ejercicio de lectoescritura digital, una estrategia que si bien aprovecha determinadas competencias de creación de producciones escritas y del uso de software y aplicaciones de escritura, termina dejando de lado muchas otras competencias transmedia que podrían resultar provechosas. Los jóvenes tienen la capacidad de escoger aquellos dispositivos que mejor se adaptan a las tareas que quieren desarrollar, por lo que, al persistir las actividades de escritura digital, terminan eligiendo la computadora de escritorio para realizarlas, motivados por la comodidad que les proporciona el teclado por sobre la escritura en pantallas. Lo importante de este punto es pensar en actividades que expandan el aprovechamiento de otras competencias transmedia y que les permitan a los estudiantes incorporar y pensar al celular como dispositivo dentro de los espacios educativos.

Otra cuestión surge de la necesidad de proponer actividades escolares que consideren las los usos y prácticas en entornos digitales. Si bien las competencias transmedia no se presentan de forma equilibrada, la propuesta pasa por promover entornos de producción digital para los jóvenes. Pensar en espacios más cercanos a las realidades de los jóvenes nos abre la posibilidad de desarrollar múltiples actividades, aprovechando el potencial abierto y flexible, además del espíritu participativo y colaborativo, que ofrecen los escenarios digitales. Creemos que las competencias transmedia que posee cada actor dentro del aula no son un limitante para la utilización de las tecnologías digitales, dado que las estrategias de aprendizaje informal permiten reconocer procesos de desarrollo de competencias transmedia. De esta forma, aquellos estudiantes que poseen determinada competencia pueden tomar el rol de expertos y colaborar con los compañeros que no tengan estas habilidades y que también podrían aprender mediante otras estrategias, como la práctica o la simulación, entre otras. Desde este enfoque, el profesor ya no tiene la responsabilidad de poseer todo el conocimiento, sino que tiene la oportunidad de aprender a la par de sus estudiantes, de generar y gestionar estos espacios digitales participativos. Aquí es interesante rescatar la noción de los docentes como influencers del aprendizaje que propone Carmen Marta Lazo (como se citó en Canal Audiovisual UNIA, 2020). Por lo tanto, la posibilidad de experimentación, del ensayo/error, que proporcionan los usos de las tecnologías digitales es fundamental para aprovechar los alfabetismos transmedia dentro de las aulas. 


\section{Austral @municación \\ Volumen 10, número 2 (Diciembre de 2021): 375-394 ISSN (I) 2313-9129. ISSN (E) 2313-9137}

Lo más relevante en esta investigación exploratoria lo encontramos en la identificación de distintas estrategias de aprendizaje informal, hallazgo que permite entender procesos de apropiación social de las tecnologías. Para esto, fue conveniente alejarnos de la noción "nativos digitales" y considerar a los jóvenes como actores que desarrollan estrategias de aprendizaje de habilidades y competencias transmedia en su relación habitual con las tecnologías digitales. Pensar en la inclusión de los alfabetismos transmedia dentro del espacio educativo no implica la sustitución o rechazo de estrategias de aprendizaje formal, sino que plantea la necesidad de poner en valor los usos y prácticas que los jóvenes tienen de las tecnologías digitales en sus vidas cotidianas para que la inclusión de las TIC no surja de una implementación puramente instrumental. Los hallazgos y reflexiones resultantes de esta investigación aportan un conocimiento situado sobre un fenómeno poco estudiado en esta región del país, brindando pistas para el desarrollo de futuras investigaciones. En este sentido, el desafío está en la necesidad de continuar actualizando las indagaciones sobre este tema, dado que el avance tecnológico, como los usos y prácticas de los jóvenes, van cambiando y transformándose constantemente.

\section{Referencias}

Albarello, F. (2018). Habilidades transmedia para el estudio: prácticas de lectura en estudiantes universitarios. Transmedia Literacy Conference. Universitat Pompeu Fabra.

Albarello, F. y Mihal, I. (2018). Del canon al fandom escolar: \#Orson80 como narrativa transmedia educativa. Comunicación y Sociedad, (33), 223-247. https://doi. org/10.32870/cys.v0i33.7055.

Almirón, M. E. (2014). La situación de las TIC en la educación argentina: un estudio de casos en dos escuelas bonaerenses (Tesis de doctorado). Universidad Nacional de Quilmes. Repositorio Institucional Digital de Acceso Abierto de la Universidad Nacional de Quilmes. https://ridaa.unq.edu.ar/handle/20.500.11807/101.

André, M. (1998). Etnografia da prática escolar. Papirus Editora.

Bernete, F. (2010). Usos de las TIC, Relaciones sociales y cambios en la socialización de las y los jóvenes. Revista de Estudios de juventud, (88), 97-114. http://www.injuve. es/sites/default/files/RJ88-08.pdf.

Black, J., Castro, J. y Lin, C. C. (2015). Youth Practices in Digital Arts and New Media: Learning in Formal and Informal Settings. Palgrave.

Bordón, E. I. (2020). Consumo de medios digitales salteños. Uso de competencias transmedia al informarse en tiempos de pandemia. En Flores, J., Oyarce, J. y Rodríguez-Garay, G. (Orgs.), Reflexões sobre internet, tecnologia e comunicação (pp. 41-61). Ria Editorial. 
Esteban Ismael Bordón

Pensar los alfabetismos transmedia dentro del aula. Tensiones entre la lógica escolar y las prácticas juveniles

Buckingham, D. (2008). Más allá de la tecnología. Aprendizaje infantil en la era de la cultura digital. Manantial Ediciones.

Canal Audiovisual UNIA. (2020). Los docentes en la era TIC: Influencers del aprendizaje. Carmen Marta Lazo (webinar V Congreso Alfamed) [Archivo de Video]. Vimeo. https://vimeo.com/466475981.

Castells, M. (2005). La Era de la Información: economía, sociedad y cultura. Siglo XXI.

Castells, M., Fernández-Ardevol, M., Qiu, J. y Sey, A. (2007). Comunicación móvil y Sociedad. Ariel.

Chartier, R. (1999). Cultura escrita, literatura e historia. Fondo de Cultura Económica.

Clark, W., Logan, K., Luckin, R., Mee, A. y Oliver, M. (2009). Beyond Web 2.0: Mapping the Technology Landscapes of Young Learners. Journal of Computer Assisted Learning, 25(1), 56-69.

Corona Rodríguez, J. (2018). De la alfabetización a los alfabetismos: aprendizaje y participación DIY de Fansy Makers mexicanos. Comunicación y Sociedad, (33), 139-169. https://doi.org/10.32870/cys.v0i33.7073.

Cuñat Giménez, R. (2007). Aplicación de la teoría fundamentada (Grounded Theory) al estudio del proceso de creación de empresas. Decisiones basadas en el conocimiento $y$ en el papel social de la empresa, (2), 1-13. http://dialnet.unirioja.es/servlet/ articulo? codigo $=2499458$.

Dávila León, O. (2004). Adolescencia y juventud: De las nociones a los abordajes. Última década, 12(21) 83-104. https://dx.doi.org/10.4067/S0718-22362004000200004.

García Canclini, N. (2007). Lectores, espectadores e internautas. Gedisa.

García Canclini, N. (2012a). Comunicación y Ciencias Sociales: el giro transdiciplinario y la política. Oficios Terrestres, 1(27). https://perio.unlp.edu.ar/ojs/index.php/ oficiosterrestres/article/view/1298.

García Canclini, N. (2012b). Geopolítica de la industria cultural e iniciativas emergentes. III Congreso Internacional de la Asociación Argentina de Estudios de Cine y Audiovisual. Córdoba, Argentina, 10 al 12 de mayo. http://www.asaeca.org/aactas/ garc_a_canclini_n_stor_-_geopol_tica_de_la_industria_cultural_e_iniciativas_ emergentes.pdf.

Huergo, J. y Fernández, M. (2000). Cultura Escolar, Cultura Mediática/Intersecciones. Universidad Pedagógica Nacional.

Jenkins, H. (2010). Convergence Culture. Where old and new media collide. University Press. Manovich, L. (2013). El software toma el mando. Editorial UOC.

Margulis, M. y Urresti, M. (1998). La construcción social de la condición de juventud. En Laverde Toscano, M., Valderrama, C. y Cubides Cipagauta, H. (Eds.), Viviendo a toda. Jóvenes, territorios culturales y nuevas sensibilidades (pp. 3-21). Universidad Central/Siglo del Hombre Editores. 
Austral Comunicación

Volumen 10, número 2 (Diciembre de 2021): 375-394 ISSN (I) 2313-9129. ISSN (E) 2313-9137

Martín-Barbero, J. (2006). Jóvenes: comunicación e identidad. Pensar Iberoamérica. https://red.pucp.edu.pe/wp-content/uploads/biblioteca/081011.pdf.

Martín-Barbero, J. (2009). Cuando la tecnología deja de ser ayuda didáctica para convertirse en mediación cultural. Teoría de la Educación. Educación y Cultura en la Sociedad de la Información, 10(1), 19-31. https://www.redalyc.org/ pdf/2010/201018023002.pdf.

Martínez Rodríguez, J. (2011). Métodos de investigación cualitativa. Silogismo, (8), 2738. https://nanopdf.com/download/metodos-de-investigacion-cualitativa_pdf.

Mason, J. (2006). Mixing methods in a qualitatively driven way. Qualitative Research, 6(1), 9-26. https://doi.org/10.1177/1468794106058866.

Pink, S. y Ardevol, E. (2018). Estrategias etnográficas para revelar prácticas transmedia de los adolescentes. En Scolari, C. (Ed.), Adolescentes, medios de comunicación y cultura colaborativa. Aprovechando las competencias transmedias de los jóvenes en el aula (pp. 114-124). Universitat Pompeu Fabra. https://repositori.upf.edu/ handle $/ 10230 / 34245$ ? localeattribute $=$ en.

Piscitelli, A. (2009). Nativos digitales. Dieta cognitiva, inteligencia colectiva y arquitecturas de la participación. Santillana.

Prensky, M. (2001). Digital Natives Digital Immigrants Part 1. On the Horizon, 9(5), $1-6$.

Puggi, M. F. (2020). La formación docente para la inclusión de tecnologías en la educación superior (Trabajo final integrador). Universidad Nacional de Quilmes. Repositorio Institucional Digital de Acceso Abierto de la Universidad Nacional de Quilmes. http://ridaa.unq.edu.ar/handle/20.500.11807/2201.

Reguillo, R. (2003). Emergencia de culturas juveniles. Estrategias del desencanto. Grupo Editorial Norma.

Scolari, C. (2015). Ecología de medios. Entornos, evoluciones e interpretaciones. Gedisa.

Scolari, C. (2018a). Introducción: del alfabetismo mediático al alfabetismo transmedia. En Scolari, C. (Ed.), Adolescentes, medios de comunicación y culturas colaborativas. Aprovechando las competencias transmedia de los jóvenes en el aula (pp. 14-23). Universitat Pompeu Fabra. https://repositori.upf.edu/ handle $/ 10230 / 34245$ ? localeattribute $=$ en.

Scolari,C.(2018b).Estrategias de aprendizaje informal.En Scolari,C.(Ed.),Adolescentes, medios de comunicación y culturas colaborativas. Aprovechando las competencias transmedia de los jóvenes en el aula (pp. 83-91). Universitat Pompeu Fabra. https:// repositori.upf.edu/handle/10230/34245?localeattribute=en.

Scolari, C. (2018c). Alfabetismo transmedia en la nueva ecología de los medios. http:// transmedialiteracy.upf.edu/sites/default/files/files/TL_whit_es.pdf.

Scolari, C., Winocur, R., Pereira, S. y Barreneche, C. (2018). Alfabetismo transmedia. 
Pensar los alfabetismos transmedia dentro del aula. Tensiones entre la lógica escolar y las prácticas juveniles

Una introducción. Comunicación y Sociedad, (33), 7-13.http://www.scielo.org.mx/ pdf/comso/n33/0188-252X-comso-33-7.pdf.

Strauss, A. y Corbin, J. (2002). Bases de la investigación cualitativa. Técnicas y procedimientos para desarrollar la teoría fundamentada. Universidad de Antioquia.

Valles Martínez, M. (1999). Técnicas cualitativas de investigación social: reflexión metodológica y práctica profesional. Síntesis.

Winocur, R., Gutiérrez, G. y Barreneche, C. (2018). Habilidades transmedia de los adolescentes y desafíos pedagógicos. Educación y Ciudad, (35), 169-178. https:// dialnet.unirioja.es/descarga/articulo/6702432.pdf. 